\section{CNS Spectrums}

www.cambridge.org/cns

\section{Original Research}

Cite this article: Dell'Osso L, Cremone IM, Chiarantini I, Arone A, Massimetti G, Carmassi C, and Carpita B (2022). Autistic traits and camouflaging behaviors: a cross-sectional investigation in a University student population. CNS Spectrums 27(6), 740-746. https://doi.org/10.1017/S1092852921000808

Received: 21 July 2021

Accepted: 30 August 2021

\section{Key words:}

Camouflaging; autism spectrum; CAT-Q; AdAS Spectrum; sex; students

\section{Author for correspondence:}

*Barbara Carpita, MD

Email: barbara.carpita1986@gmail.com (c) The Author(s), 2021. Published by Cambridge University Press. This is an Open Access article, distributed under the terms of the Creative Commons Attribution licence (http:// creativecommons.org/licenses/by/4.0/), which permits unrestricted re-use, distribution, and reproduction in any medium, provided the original work is properly cited.

\title{
Autistic traits and camouflaging behaviors: a cross-sectional investigation in a University student population
}

\section{Liliana Dell’Osso, Ivan M. Cremone, Ilaria Chiarantini, Alessandro Arone, Gabriele Massimetti, Claudia Carmassi (i) and Barbara Carpita* (i)}

\section{Department of Clinical and Experimental Medicine, University of Pisa, Pisa, Italy}

\section{Abstract}

Background. Increasing research is stressing the importance of identifying autistic traits (ATs) in clinical and general populations. University students may be a group at higher risk for the presence of ATs. Recently, specific attention has been paid to camouflaging strategies used by subjects in the autism spectrum in order to cope with the social environment. The aim of this work was to evaluate the prevalence of ATs and camouflaging behaviors in a population of University students.

Methods. Subjects were requested to anonymously fill out through an online form the Adult Autism Subthreshold Spectrum and the Camouflaging AT Questionnaire.

Results. ATs were more represented among males and among students of specific fields of study. Camouflaging behaviors were significantly more frequent among subjects with more severe autism spectrum symptoms, without differences depending from sex.

Conclusions. Our study confirms the strong association between ATs and camouflaging behaviors and the relationship between ATs, sex, and specific fields of study.

\section{Introduction}

Autism spectrum is a psychopathological dimension characterized by an impairment in verbal and nonverbal communication, narrow interests, and stereotyped behaviors, which can occur in different degrees of severity: from subthreshold forms and autistic traits (ATs) to full-blown autism spectrum disorder (ASD). ${ }^{1-3}$ In psychopathology, the spectrum model allows a more accurate description of different mental disorders, including ASD, including not only fullfledged symptoms, but also personality traits, atypical and isolated manifestations, prodromal clinical features, and behavioral characteristics which may be present before, during, or after the onset of the core symptoms of the full-blown disorder. ${ }^{3-5}$ Several researches suggested the presence of comorbidity between ASD and other psychiatric disorders, such as anxiety and mood disorders. ${ }^{3,5-7}$ Recent researches stressed how autism spectrum may represent a condition of vulnerability that may lead to develop other psychopathological conditions. ${ }^{8,9}$ In this framework, increasing literature is stressing how subthreshold ATs seem to be distributed in a continuum from the clinical to the general population, being more represented in specific high-risk group, and in particular among clinical sample of subjects with other psychiatric conditions. ${ }^{3,5-7,10,11}$ Students with ATs may face stressful consequences once they enter University, such as difficulties in adjusting to a different life style and rhythm, as well as to different class, lecture, and exam requirements. ${ }^{12}$ The creation and maintenance of a stable social network may also be compromised, along with taking part in group projects for academic tasks and, eventually, sharing common spaces in University accommodations. ${ }^{13-17}$ Although, especially when concerning subthreshold ATs, some students may reach an adjustment to the new social context and occupation on their own, depending on their personality and cognitive style, authors are stressing that an adequate support to students with ASD or ATs is strongly needed. ${ }^{18-20}$ Previous studies pointed out to an important role of gender, since male students present more frequently ATs than female students. ${ }^{18,21-23}$ Moreover, high-ranking University students, who were also reported to possibly be at higher risk to show ATs, may be exposed to greater environmental stress. ${ }^{3,18}$ Some authors highlighted an association between the kind of University course chosen and the presence of ATs, generally stressing a higher presence of ATs among students of scientific courses. ${ }^{18,21-24}$ In the last years, the need of understanding and detecting milder forms of ASD and ATs, in order to improve the quality of life of the subjects, lead to an increasing interest toward the camouflaging behaviors often used by individuals in the autism spectrum. ${ }^{25,26}$ Camouflaging is considered one of the strategies that subjects with ASD use to mask their symptoms in front of others and to adjust to the social environment. ${ }^{25,26}$ It may feature the use of clichés, jokes, or previously learned phrases, as well as the imitation of gestures 
and expressions used by other people. ${ }^{26}$ On the other hand, the repeated use of these strategies in every social context was reported to be very stressful for the subjects, implying continuous efforts to hyperadapt to the environment and often leading to anxiety or depressive symptoms. ${ }^{26}$ Self-identity and self-perception may be damaged, forcing the subjects to build a mask in order to interact with others, ${ }^{25}$ whereas some of their actual needs may be overlooked or misunderstand. ${ }^{27}$ These strategies seem to be widely present among autistic people, with a rate of estimated prevalence up to $70 \% .{ }^{28}$ Hull et al $^{29}$ recently developed and validated the first self-report questionnaire for measuring camouflaging behaviors among subjects in the autism spectrum, the Camouflaging AT Questionnaire (CAT-Q), which explores different dimensions of camouflaging. In the framework of the increasing interest toward ASD presentations among females, previous literature has described camouflaging as a behavior more often associated with female gender, hypothesizing also a link between the use of these strategies and the underdiagnosis of ASD among women. ${ }^{25-27,29,30}$ According to these authors, female ASD phenotypes would feature, together with specific kinds of narrow interests, a milder impairment in social interaction, also due to a higher awareness of their own social difficulties, which would lead to a greater use of camouflaging strategies. ${ }^{25-27,29,30}$ This hypothesis would be somewhat in line with the higher prevalence of social anxiety reported among subjects with high-functioning autism, who often show a better understanding of their difficulties in social interactions: noticeably, social anxiety is a condition with a higher prevalence among women and which is associated, like ASD, with an impairment of the social brain. ${ }^{9,31,32,33}$ Despite that, recent studies that explored gender differences in camouflaging with the CAT-Q led to controversial results. Hull et al reported higher camouflaging tendencies in ASD females than in ASD males, but they did not find gender differences in CAT-Q scores among typically developed subjects. ${ }^{35}$ Moreover, another study did not find gender differences in CAT-Q scores in a sample of ASD subjects, although it highlighted that males and females may use camouflaging for different reasons. ${ }^{28,36}$ It was also suggested that gender differences in camouflaging behaviors may depend on sociocultural factors. ${ }^{37}$ Some authors by means of CAT-Q confirmed among ASD subjects a link between camouflaging strategies and neuroticism, ${ }^{38}$ anxiety, and depressive symptoms, ${ }^{39}$ whereas other stressed a greater use of camouflaging strategies among ASD subjects with less impaired executive functions. ${ }^{40}$ Noticeably, the link between CAT-Q scores and psychological distress was reported also in nonclinical samples. ${ }^{41}$ Moreover, in a sample of University students, a positive correlation was found between CAT-Q scores and suicidal behaviors, with camouflaging partially mediating the relationship between ATs, thwarted belongingness and suicidality. ${ }^{42}$

In this framework, the aim of the present study was to assess the prevalence of ATs and camouflaging behaviors in a population of University students.

\section{Methods}

The present study was conducted among University students at the University of Pisa by the Department of Clinical and Experimental Medicine, section of Psychiatry, in collaboration with the local University Institutional Governance (Rectorate). All the students received an invitation to participate via email, and they were requested to anonymously fulfill a brief form for sociodemographic variables and to provide their responses to the psychometric questionnaires. As explained in the instructions provided by email, participants who fulfilled the questionnaires automatically gave their consent to be enrolled in the study. Subject did not receive any payment/benefit for participating in the survey. The Athenaeum Ethical Committee approved all the procedures.

\section{Instruments}

\section{The Adult Autism Subthreshold Spectrum}

The Adult Autism Subthreshold Spectrum (AdAS Spectrum) is an instrument developed and validated by Dell'Osso et $\mathrm{al}^{43}$ with the aim to assess the wide range of autism spectrum manifestations among adults with average intelligence and without language development alterations. The questionnaire was validated in Italian, English, and Spanish, reporting good validity and reliability features. ${ }^{43,44}$ It is composed by 160 questions with dichotomous answers (yes/no), organized in seven domains: Childhood/Adolescence, Verbal Communication, Nonverbal Communication, Empathy, Inflexibility and Adherence to Routine, Restricted Interests and Rumination, and Hyper/Hyporeactivity to Sensory Input. A higher score to the questionnaire indicates higher autistic features. Two cutoff scores were identified for this instrument: scoring over 43 suggests the presence of significant ATs, whereas scoring over 70 indicates a high risk for the presence of full-threshold ASD symptoms. $^{45}$

\section{Camouflaging AT Questionnaire}

The CAT-Q is a self-report questionnaire recently developed and validated in English by Hull et al. ${ }^{29}$ This instrument is tailored to evaluate social camouflaging behaviors from a quantitative point of view. It is composed by 25 items, with answers organized on a seven-point Likert scale, and it is divided in three subscales: Compensation (measuring the use of actively compensative behaviors), Masking (measuring the habit of hiding autistic-like features), and Assimilation (measuring the attempts to fit in with others). Recently, Dell'Osso et al validated an Italian version of the instrument, which demonstrated excellent internal consistency and test-retest reliability, showing also strong correlations with other instruments assessing the autism spectrum. ${ }^{46}$

\section{Statistical analyses}

In order to perform comparisons among students of different academic fields, we grouped the academic courses in four major areas, as follows: Pure Sciences (Biology, Chemistry, Mathematics, Physics, Informatics, and Earth Sciences); Applied Sciences (Engineering, Health Sciences, Veterinary Sciences, Pharmacy, and Agricultural Sciences); Humanities (Modern and Ancient Humanities, Historical Science, Philosophy, Foreign Language and Literature, and Digital Humanities); and Economics, Law, and Political sciences.

A two-way multivariate analysis of variance (MANOVA) analysis was performed to evaluate the effect of gender and academic field on the score reported on AdAS Spectrum domains. Student's $t$-test or analysis of variance (ANOVA) analyses (followed by Bonferroni's post hoc tests) were performed in order to compare AdAS Spectrum total and domain scores in the subgroups defined by the variables for which the MANOVA analysis reported a significant effect on AdAS Spectrum scores. In the subgroup of students who reported scores beyond the subthreshold for ATs 
according to AdAS Spectrum, we performed a three-way MANOVA in order to evaluate the effect of gender, presence of AT/ASD clinical symptoms, and academic fields of study on CAT-Q domain scores. Student's $t$-test or ANOVA analyses were performed in order to compare CAT-Q total and domain scores in the subgroups defined by the variables for which the MANOVA analysis reported a significant effect on CAT-Q scores.

\section{Results}

A total of 2141 students were enrolled in the study. Female were $66.10 \%(n=1415)$ of the total sample, whereas males were $33.90 \%$ $(\mathrm{n}=726)$. The mean score reported on AdAS Spectrum was $51.291 \pm 23.641$. The two-way MANOVA analysis, conducted with sex and academic field as independent variables and AdAS Spectrum domain scores as dependent variables, highlighted a significant main effect of both academic field $(P<.001)$ and sex $(P<.001)$ on AdAS Spectrum domain scores. However, no significant effect was found for interaction gender ${ }^{\star}$ sex (see Table 1 ). In particular, "Pure sciences" students showed significant higher AdAS Spectrum scores on all domains when compared with the "Applied Sciences" group. Moreover, the "Pure Sciences" group

Table 1. Two-Way MANOVA Analysis: Adult Autism Subthreshold Spectrum Domain Scores as Dependent Variables, and Sex and Academic Field as Independent Variables

\begin{tabular}{lccc}
\hline Effect & Pillai's Trace & $F$ & $P$ \\
\hline Intercept & 0.763 & 975.543 & $<.001$ \\
\hline Sex & 0.014 & 4.353 & $<.001$ \\
\hline Academic field & 0.041 & 4.171 & $<.001$ \\
\hline Sex*academic field & 0.010 & 1.020 & .434 \\
\hline
\end{tabular}

The significant values of $p(p<.05)$ were highlighted in bold in the table. showed also significantly higher scores than the "Economics, Law, and Political Sciences" group on AdAS Spectrum Childhood/Adolescence and Empathy domains, whereas it scored higher than the "Humanities" group on AdAS Spectrum Verbal Communication and Empathy domains. On the other hand, the "Humanities" group scored significantly higher than the "Applied Sciences" group on AdAS Spectrum Childhood/Adolescence, Nonverbal Communication, and Restricted Interest and Rumination domains. Finally, the ANOVA analysis highlighted significant differences on AdAS Spectrum total scores depending on academic field, with students of "Pure Sciences" scoring higher than those of "Applied Sciences" and "Economics, Law, and Political Sciences," whereas students of "Humanities" scored higher than those of "Applied Sciences" (see Table 2). When considering sex differences, we found that males scored significantly higher than females on AdAS Spectrum total scores and on AdAS Spectrum Childhood/Adolescence, Verbal Communication, Empathy, and Restricted Interests and Rumination domains (see Table 3). In the subgroups of students scoring beyond the subthreshold for ATs according to AdAS Spectrum, a three-way MANOVA analysis was performed with sex, academic field, and presence of AT/ASD clinical symptoms as independent variables and CAT-Q domain scores as dependent variables. The MANOVA analysis highlighted a significant main effect of both academic field $(P<.001)$ and presence of AT/ASD clinical symptoms $(P<.001)$ on AdAS Spectrum domain scores. However, no significant effect was found for sex and for the interactions among variables (see Table 4). Considering the academic field, we found that the "Pure Sciences" group reported higher scores than all the other groups on CAT-Q Assimilation domain, while scoring higher than "Applied Sciences" and "Humanities" on CAT-Q Compensation domain. The ANOVA analysis with the total CAT-Q score as the dependent variable showed significant differences among groups, with the "Pure Sciences" group showing significantly higher score than the "Applied Sciences" group (see Table 5). Finally, we found that subjects with subthreshold ATs scored

Table 2. Comparison of Adult Autism Subthreshold Spectrum (AdAS Spectrum) Total and Domain Scores Among Students of Different Academic Fields

\begin{tabular}{|c|c|c|c|c|c|c|c|}
\hline & $\begin{array}{l}\text { Pure Sciences } \\
\text { (Group 1) }\end{array}$ & $\begin{array}{l}\text { Applied } \\
\text { Sciences } \\
\text { (Group 2) }\end{array}$ & $\begin{array}{l}\text { Humanities } \\
\text { (Group 3) }\end{array}$ & $\begin{array}{c}\text { Economics, Law, and } \\
\text { Political Sciences (Group 4) }\end{array}$ & & & \\
\hline & $\mathrm{n}=500$ & $\mathrm{n}=949$ & $\mathrm{n}=502$ & $\mathrm{n}=190$ & & & Bonferroni Post \\
\hline & Mean $\pm S D$ & Mean \pm SD & Mean $\pm S D$ & Mean $\pm S D$ & $F$ & $P$ & Hoc Comparisons \\
\hline $\begin{array}{l}\text { AdAS Spectrum total } \\
\text { score }\end{array}$ & $55.344 \pm 24.217$ & $48.505 \pm 22.936$ & $53.358 \pm 23.653$ & $49.078 \pm 23.657$ & 11.283 & $<.001$ & $\begin{array}{l}\text { Group } 1>\text { Group } \\
2,4 ; \text { Group } 3>2\end{array}$ \\
\hline $\begin{array}{l}\text { AdAS Childhood/ } \\
\text { Adolescence }\end{array}$ & $8.162 \pm 4.138$ & $6.817 \pm 3.853$ & $7.918 \pm 3.952$ & $6.531 \pm 3.875$ & 18.990 & $<.001$ & $\begin{array}{l}\text { Group } 1,3> \\
\text { Group 2,4 }\end{array}$ \\
\hline $\begin{array}{l}\text { AdAS Verbal } \\
\text { Communication }\end{array}$ & $6.140 \pm 3.318$ & $5.20 \pm 3.104$ & $5.567 \pm 3.215$ & $5.042 \pm 3.148$ & 10.813 & $<.001$ & $\begin{array}{l}\text { Group } 1> \\
\text { Group 2,3,4 }\end{array}$ \\
\hline $\begin{array}{l}\text { AdAS Nonverbal } \\
\text { Communication }\end{array}$ & $10.556 \pm 4.920$ & $9.381 \pm 4.831$ & $10.577 \pm 4.971$ & $9.494 \pm 4.977$ & 10.105 & $<.001$ & $\begin{array}{c}\text { Group } 1,3> \\
\text { Group } 2\end{array}$ \\
\hline AdAS Empathy & $3.334 \pm 2.383$ & $2.915 \pm 2.282$ & $2.904 \pm 2.285$ & $2.836 \pm 2.179$ & 4.552 & .003 & $\begin{array}{c}\text { Group } 1>\text { Group 2; } \\
\text { Group } 3\end{array}$ \\
\hline $\begin{array}{l}\text { AdAS Inflexibility and } \\
\text { Adherence to Routine }\end{array}$ & $14.054 \pm 7.0539$ & $12.631 \pm 6.660$ & $13.513 \pm 7.178$ & $13.215 \pm 7.377$ & 4.981 & .002 & $\begin{array}{r}\text { Group } 1> \\
\text { Group } 2\end{array}$ \\
\hline $\begin{array}{l}\text { AdAS Restricted } \\
\text { Interests and } \\
\text { Rumination }\end{array}$ & $8.766 \pm 4.476$ & $7.678 \pm 4.276$ & $8.338 \pm 4.433$ & $8.131 \pm 4.450$ & 7.265 & $<.001$ & $\begin{array}{c}\text { Group } 1,3> \\
\text { Group } 2\end{array}$ \\
\hline $\begin{array}{l}\text { AdAS Hyper/ } \\
\text { Hyporeactivity to } \\
\text { Sensory Input }\end{array}$ & $4.332 \pm 3.256$ & $3.875 \pm 3.134$ & $4.537 \pm 3.317$ & $3.826 \pm 3.208$ & 5.902 & .001 & $\begin{array}{r}\text { Group } 1> \\
\text { Group } 2\end{array}$ \\
\hline
\end{tabular}

The significant values of $p(p<.05)$ were highlighted in bold in the table. 
Table 3. Comparison of Adult Autism Subthreshold Spectrum (AdAS Spectrum) Total and Domain Scores Between Males and Females

\begin{tabular}{|c|c|c|c|c|}
\hline & Males & Females & & \\
\hline & $\mathrm{n}=726$ & $\mathrm{n}=1411$ & & \\
\hline & Mean \pm SD & Mean \pm SD & $T$ & $P$ \\
\hline AdAS Spectrum total score & $53.170 \pm 19.451$ & $50.327 \pm 23.153$ & 2.638 & .008 \\
\hline AdAS Childhood/Adolescence & $7.869 \pm 3.993$ & $7.105 \pm 3.974$ & 4.203 & $<.001$ \\
\hline AdAS Verbal Communication & $5.798 \pm 3.357$ & $5.337 \pm 3.117$ & 3.156 & .002 \\
\hline AdAS Nonverbal Communication & $10.085 \pm 4.875$ & $9.874 \pm 4.957$ & 0.935 & .350 \\
\hline AdAS Empathy & $3.385 \pm 2.492$ & $2.807 \pm 2.176$ & 5.297 & $<.001$ \\
\hline AdAS Inflexibility and Adherence to Routine & $13.487 \pm 7.085$ & $13.086 \pm 6.895$ & 1.263 & .207 \\
\hline AdAS Restricted Interests and Rumination & $8.469 \pm 4.560$ & $7.951 \pm 4.299$ & 2.535 & .011 \\
\hline AdAS Hyper/Hyporeactivity to Sensory Input & $4.074 \pm 3.271$ & $4.163 \pm 3.200$ & -0.604 & .546 \\
\hline
\end{tabular}

The significant values of $p(p<.05)$ were highlighted in bold in the table.

Table 4. Three-Way MANOVA Analysis: CAT-Q Domain Scores as Dependent Variables, Sex, Presence of Autistic Trait/Autism Spectrum Disorder Clinical Symptoms According to Adult Autism Subthreshold Spectrum (AdAS Spectrum; "AdAS Spectrum Threshold"), and Academic Field as Independent Variables

\begin{tabular}{|c|c|c|c|}
\hline Effect & Pillai's Trace & $F$ & $P$ \\
\hline Sex & 0.005 & 2.350 & .071 \\
\hline AdAS Spectrum threshold & 0.146 & 73.908 & $<.001$ \\
\hline Academic field & 0.030 & 4.435 & $<.001$ \\
\hline Sex*academic field & 0.005 & 0.776 & .639 \\
\hline AdAS Spectrum threshold*academic field & 0.008 & 1.223 & .276 \\
\hline Sex*academic field*AdAS Spectrum threshold & 0.005 & 0.736 & .676 \\
\hline
\end{tabular}

The significant values of $p(p<.05)$ were highlighted in bold in the table.

Table 5. Comparison of CAT-Q Total and Domain Scores Among Students of Different Academic Fields

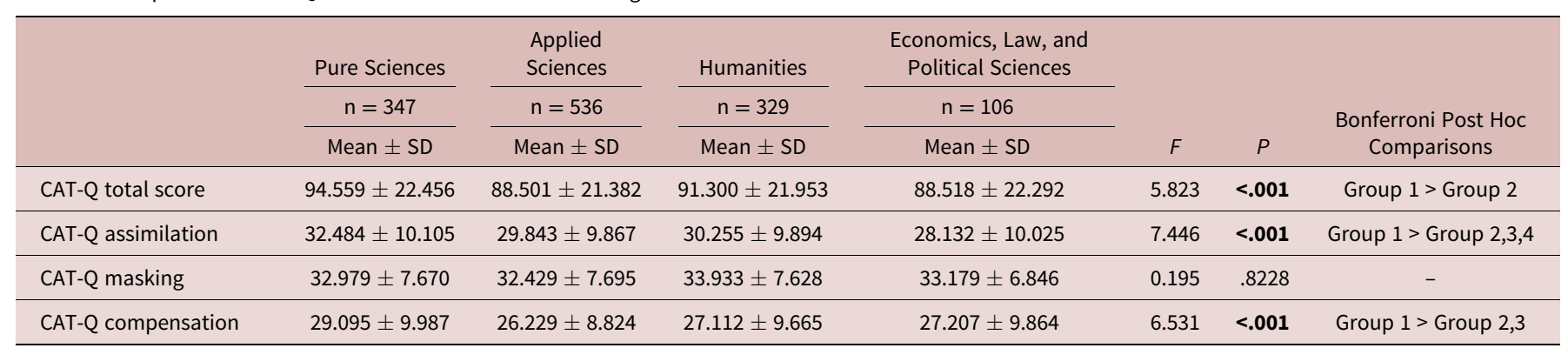

The significant values of $p(p<.05)$ were highlighted in bold in the table.

significantly lower on CAT-Q total and domain scores than subjects with ASD clinical symptoms (see Table 6).

\section{Discussion}

The aim of the present work was to evaluate the presence of ATs and camouflaging behaviors in a population of University students. Concerning ATs, we found in our sample a mean AdAS Spectrum score of around 51, which is in the medium range of the questionnaire for the risk of ATs. This result, which suggests a significant presence of ATs among University students, is in line with previous research pointing out a higher frequency of ATs in University populations, in particular in those Universities with selective entrance exams or ultracompetitive study paths. ${ }^{47}$

Our study highlighted also a higher AdAS Spectrum total score among male students $(53.170 \pm 19.451)$ than female ones $(50.327 \pm 23.153)$. Males scored higher than females also in most of AdAS Spectrum domains. These data suggest that, in the present population, male sex was more frequently associated with ATs than female sex. Such a result is consistent with a large amount of previous researches, ${ }^{18,21-23}$ which stressed an association between male sex and both ASD and ATs, and it is also in line with the "Extreme Male Brain Theory of Autism" proposed by BaronCohen et al, which hypothesized that autistic individuals may show male-typical brain features of mental functioning, such as reduced 
Table 6. Comparison of CAT-Q Total and Domain Scores Between Students with Subthreshold Autistic Trait (AT Group) and Autism Spectrum Disorder (ASD) Clinical Symptoms (ASD-Like Group)

\begin{tabular}{|c|c|c|c|c|}
\hline & AT Group & ASD-Like Group & & \\
\hline & $n=867$ & $n=451$ & & \\
\hline & Mean \pm SD & Mean \pm SD & $T$ & $P$ \\
\hline CAT-Q total score & $83.576 \pm 18.842$ & $104.676 \pm 20.986$ & 18.540 & $<.001$ \\
\hline CAT-Q assimilation & $27.382 \pm 8.895$ & $36.503 \pm 9.3219$ & 17.371 & $<.001$ \\
\hline CAT-Q masking & $31.699 \pm 7.416$ & $35.5299 \pm 7.381$ & 8.911 & $<.001$ \\
\hline CAT-Q compensation & $24.494 \pm 8.037$ & $32.643 \pm 9.791$ & 16.174 & $<.001$ \\
\hline
\end{tabular}

The significant values of $p(p<.05)$ were highlighted in bold in the table.

empathy skills and better systemizing. ${ }^{48}$ However, it should be noted that, in line with previous studies in similar populations, which did not find gender differences with respect to ATs, ${ }^{10}$ the score difference between males and females was limited (less than three points), with both sex scoring in the medium range of risk for ATs. Recent literature stressed the importance of reconsidering gender differences in the autism spectrum in light of the growing understanding about the underestimation of autism spectrum symptoms among females. ${ }^{29,45}$ However, it is possible that, even with instruments tailored to assess gender-specific symptoms such as the AdAS Spectrum, ATs would remain slightly more represented among males, although with lower gender gaps than those reported in the past. ${ }^{18,45}$ Furthermore, we found significant differences in ATs among students of different academic fields. In particular, students of "Pure Sciences" showed a mean AdAS Spectrum score significantly higher than students of "Applied Sciences" and of "Economics, Law, and Political Sciences." Moreover, students of "Pure Sciences" reported also higher scores on several AdAS Spectrum domains with respect to students of other academic fields. This result is in line with previous findings, which stressed an association between studying in the science field and the presence of higher ATs, ${ }^{18,21,23}$ leading also to hypothesize a link between ATs and scientific skills. ${ }^{18}$ Despite that, we did not find significant differences on AdAS Spectrum total scores and on most of AdAS Spectrum domain scores between students of "Pure Sciences" and students of "Humanities," who reported significantly lower scores than the first ones only on AdAS Spectrum Verbal Communication and Empathy domains. Students of "Humanities" instead reported higher scores than students in the field of "Applied Sciences" on AdAS Spectrum total and on three AdAS Spectrum domains. These data are in contrast to previous researches that reported a strongly higher prevalence of ATs among students of sciences than among students of "Humanities." 18,21,23,49 However, they are also somewhat in line with a previous study, which stressed the presence of intermediate levels of ATs in students of "Humanities": these latters reported lower levels of ATs, measured by the Autism-Spectrum Quotient, than students of sciences but higher levels than students of medicine and of social sciences. ${ }^{24}$ As previously hypothesized by Pisula et al, ${ }^{24}$ a possible explanation of these findings may be found considering that students who choose the "Humanities" field, and especially some specific fields such as Classics and Linguistic studies, may have some ATs such as a greater interest in systems and systematization, and/or in an analytic approach toward their matter of study. ${ }^{24,48}$ In this framework, it should also be noted that most of the previous researches did not differentiate between students of theoretical and applied field of sciences. We found that students of "Humanities," who have a more theoretical study path, scored at intermediate levels between students of "Pure Sciences" and the other ones, with a significantly higher score than students of "Applied Sciences." It is possible that subjects with higher ATs would find more suitable for their interests not only for scientific studies, but also for more theoretical and less practical-oriented study paths.

Our findings confirm the strong relationship between autism spectrum and camouflaging behaviors, considering the significantly higher presence of camouflaging among subjects in the more severe range of the autism spectrum. ${ }^{26,29,35,46}$ Similarly, we found that students of "Pure Sciences" reported the highest CAT-Q scores, and students of "Applied Sciences" the lowest. Since ATs seem to be distributed continuously in the population, it is possible that also camouflaging would feature a similar distribution, and should be considered as a dimensional characteristic. ${ }^{45}$ It might be also hypothesized that the general functioning of an individual may depend on a delicate interlocking between the underlying ATs and the eventual success of compensatory strategies, such as camouflaging, although limited by the distress related to it. ${ }^{45,50}$ We did not find a significant influence of sex on CAT-Q scores. Similar results were reported by a previous study, which did not find sex differences in CAT-Q scores with respect of sex, although highlighting that camouflaging behaviors may be used for different reasons by males and females. ${ }^{28,36}$ Another study by Hull et al reported sex differences in CAT-Q scores only among ASD subjects, but not among typically developed ones. ${ }^{38}$ On the other hand, this finding differs from those reported by other studies in this field, which highlighted a greater prevalence of camouflaging behaviors among females, suggesting also a specific link between camouflaging and female autism phenotypes. ${ }^{27,29,35}$ However, it should be noted that discrepancies in sex distribution of camouflaging behaviors may vary depending on cultural factors and on the specific population chosen. In the case of our study, given that following a University course may require a certain level of communication skills in order to adjust to the environment (eg, taking oral examination, ask questions, and interact with other students), it might be hypothesized that, among subjects with ATs, only those with higher camouflaging skills would decide to enroll to University and/or would not drop out from their academic career. In this framework, it is possible that we did not find sex difference in our sample with respect to camouflaging behaviors due to the specific population examined. The hypothesis that males with ATs but lower camouflaging abilities would renounce to choose an academic path might also be related, eventually, with the higher prevalence of female students in University courses, ${ }^{51}$ although further studies are needed in order to clarify the relationship between camouflaging, sex and higher education.

This study should be considered in light of several limitations. First, the cross-sectional design of the study did not allow us to 
make inferences about casual/temporal relationships among the observed variables. Second, the sample was recruited on a voluntary basis, eventually facilitating selection biases depending on the subjective interest in the research and/or other factors. As a consequence, the population could not be actually representative of University students. Moreover, the study featured only selfreport questionnaires, filled out anonymously by participants: subsequently, results may be exposed to inaccuracies, as well as be biased by subjects' judgment on their own feelings, eventually resulting in underestimation or overestimation of the reported symptoms. Globally, results from this work further confirm the association between ATs and camouflaging behaviors, and the strong presence of both of them in University populations. It should be noted that ATs are known to be a consistent risk factor for stress-related disorders. In particular, subjects with ATs seem to show a higher vulnerability toward stressful life events, and they would develop more often post-traumatic stress disorder-like symptoms also after being exposed to mild distressful situations. ${ }^{3,52-56}$ Moreover, camouflaging strategies, among subjects with ATs, were reported to be associated with greater levels of psychological distress and also suicidal ideation. ${ }^{26,29,42}$ Both ATs and stress-related disorders have been associated in the literature with mood, anxiety disorders, and suicidality, whereas a mediating role of stress-related symptoms in the relationship between ATs and mood symptoms has also been suggested. ${ }^{7}$ In this framework, it is possible that subjects with ATs, and eventually those who use more often camouflaging behaviors, may be considered a higher risk group for the onset of other psychiatric disorders. ${ }^{3,26,29,42}$ From a clinical perspective, results from this study further suggest the need of preventive strategies for mental health among University students. A better understanding of camouflaging behaviors and of specific patterns in AT distribution may help to avoid the problem of under-recognition for these conditions and to promote more subject-tailored preventive and therapeutic strategies, allowing an early identification of psychiatric symptoms and limiting the development of chronic conditions. On the other hand, from a research point of view, results from this study add to previous literature, which is stressing the link between ATs and camouflaging behaviors, along with its possible implications. However, the pattern of ATs and camouflaging distribution depending on sex and academic field reported in the present population provides new information and insights, suggesting a specific association of these conditions with some sociodemographic characteristics. These data may open the way to further studies, with standardized protocols and longitudinal design, in order to better investigate the relationship between ATs and camouflaging behaviors, as well as their distribution, depending on specific sociodemographic variables.

Disclosure. The authors have nothing to disclose.

\section{References}

1. Dell'Osso L, Dalle Luche R, Maj M. Adult autism spectrum as a transnosographic dimension. CNS Spectr. 2016;21(2):131-133.

2. Dell'Osso L, Luche RD, Gesi C, et al. From Asperger's autistischen psychopathen to DSM-5 autism spectrum disorder and beyond: a subthreshold autism spectrum model. Clin Pract Epidemiol Ment Health. 2016;12: 120-131.

3. Dell'Osso L, Lorenzi P, Carpita B. Autistic traits and illness trajectories. Clin Pract Epidemiol Ment Health. 2019;15:94-98.
4. Frank E, Cassano, GB, Shear MK, et al. The spectrum model: a more coherent approach to the complexity of psychiatric symptomatology. CNS Spectr. 1998;3(4):23-34.

5. Dell'Osso L, Cremone IM, Carpita B, et al. Rumination, posttraumatic stress disorder, and mood symptoms in borderline personality disorder Neuropsychiatr Dis Treat. 2019;15:1231-1238.

6. Dell'Osso L, Conversano C, Corsi M, et al. Polysubstance and behavioral addictions in a patient with bipolar disorder: role of lifetime subthreshold autism spectrum. Case Rep Psychiatry. 2018;2018:1547975.

7. Dell'Osso L, Carpita B, Cremone IM, et al. The mediating effect of trauma and stressor related symptoms and ruminations on the relationship between autistic traits and mood spectrum. Psychiatry Res. 2019;279:123-129.

8. Dell'Osso L, Carpita B, Bertelloni CA, et al. Subthreshold autism spectrum in bipolar disorder: prevalence and clinical correlates. Psychiatry Res. 2019 281:112605.

9. Carpita B, Carmassi C, Calderoni S, et al. The broad autism phenotype in real-life: clinical and functional correlates of autism spectrum symptoms and rumination among parents of patients with autism spectrum disorder. CNS Spectr. 2020;25(6):765-773.

10. Carpita B, Muti D, Muscarella A, et al. Sex differences in the relationship between PTSD spectrum symptoms and autistic traits in a sample of university students. Clin Pract Epidemiol Ment Health. 2019;15:110-119.

11. Carpita B, Muti D, Petrucci A, et al. Overlapping features between social anxiety and obsessive-compulsive spectrum in a clinical sample and in healthy controls: toward an integrative model. CNS Spectr. 2020;25(4): 527-534.

12. Sasaki T. Autism spectrum disorders in Japanese university students. Jpn J Psychiatric Treat. 2010;25:1647-1652.

13. Adreon D, Durocher JS. Evaluating the college transition needs of individuals with high-functioning autism spectrum disorders. Interv Sch Clin. 2007;42(5):271-279.

14. Gelbar NW, Smith I, Reichow B. Systematic review of articles describing experience and supports of individuals with autism enrolled in college and university programs. J Autism Dev Disord. 2014;44(10):2593-2601.

15. Lambe S, Russell A, Butler C, et al. Autism and the transition to university from the student perspective. Autism. 2019;23(6):1531-1541.

16. Lei J, Ashwin C, Brosnan M, et al. Developing an online tool to measure social network structure and perceived social support amongst autistic students in higher education: a feasibility study. J Autism Dev Disord. 2019;49:3526-3542.

17. Lei J, Calley S, Brosnan M, Ashwin C, Russell A. Evaluation of a transition to university programme for students with autism spectrum disorder. $J$ Autism Dev Disord. 2020;50(7):2397-2411.

18. Baron-Cohen S, Wheelwright S, Skinner R, Martin J, Clubley E. The Autism-Spectrum Quotient (AQ): evidence from Asperger syndrome/ high-functioning autism, males and females, scientists and mathematicians [published correction appears in J Autism Dev Disord 2001 Dec;31(6):603] J Autism Dev Disord. 2001;31(1):5-17.

19. Nevill RE, White SW. College students' openness toward autism spectrum disorders: improving peer acceptance. J Autism Dev Disord. 2011;41(12): 1619-1628.

20. Woodbury-Smith MR, Robinson J, Wheelwright S, Baron-Cohen S. Screening adults for Asperger Syndrome using the AQ: a preliminary study of its diagnostic validity in clinical practice. J Autism Dev Disord. 2005;35(3): $331-335$

21. Kitazoe N, Inoue S, Izumoto Y, Kumagai N, Iwasaki Y. The AutismSpectrum Quotient in university students: pattern of changes in its scores and associated factors. Asia Pac Psychiatry. 2015;7(1):105-112.

22. Kunihira Y, Senju A, Dairoku H, Wakabayashi A, Hasegawa T. Autistic traits in non-autistic Japanese populations: relationships with personality traits and cognitive ability. J Autism Dev Disord. 2006;36(4):553-566.

23. Wakabayashi A, Baron-Cohen S, Wheelwright S, Tojo Y. The AutismSpectrum Quotient (AQ) in Japan: a cross-cultural comparison. J Autism Dev Disord. 2006;36(2):263-270.

24. Pisula E, Kawa R, Szostakiewicz Ł, Łucka I, Kawa M, Rynkiewicz A. Autistic traits in male and female students and individuals with high functioning autism spectrum disorders measured by the Polish version of the AutismSpectrum Quotient. PLoS One. 2013;8(9):e75236. 
25. Hull L, Petrides KV, Allison C, et al. "Putting on my best normal": social camouflaging in adults with autism spectrum conditions. J Autism Dev Disord. 2017;47(8):2519-2534.

26. Dell'Osso L, Lorenzi P, Carpita B. Camouflaging: psychopathological meanings and clinical relevance in autism spectrum conditions. CNS Spectr. 2020:1-3. Published May 26, 2020.

27. Bargiela S, Steward R, Mandy W. The experiences of late-diagnosed women with autism spectrum conditions: an investigation of the female autism phenotype. J Autism Dev Disord. 2016;46(10):3281-3294.

28. Cage E, Troxell-Whitman Z. Understanding the reasons, contexts and costs of camouflaging for autistic adults. J Autism Dev Disord. 2019;49(5): 1899-1911.

29. Hull L, Mandy W, Lai MC, et al. Development and validation of the Camouflaging Autistic Traits Questionnaire (CAT-Q). J Autism Dev Disord. 2019;49(3):819-833.

30. Lai MC, Lombardo MV, Auyeung B, et al. Sex/gender differences and autism: setting the scene for future research. J Am Acad Child Adolesc Psychiatry. 2015;54(1):11-24.

31. Marazziti D, Abelli M, Baroni S, Carpita B, Piccinni A, Dell'Osso L. Recent findings on the pathophysiology of social anxiety disorder. Clin Neuropsychiatry. 2014;11(2):91-100.

32. Marazziti D, Abelli M, Baroni S, Carpita B, Ramacciotti CE, Dell'Osso L. Neurobiological correlates of social anxiety disorder: an update. CNS Spectr. 2015;20(2):100-111.

33. Dell'Osso L, Abelli M, Pini S, et al. Dimensional assessment of DSM-5 social anxiety symptoms among university students and its relationship with functional impairment. Neuropsychiatr Dis Treat. 2014;10:1325-1332.

34. Dell'Osso L, Abelli M, Pini S, et al. The influence of gender on social anxiety spectrum symptoms in a sample of university students. Riv Psichiatr. 2015; 50(6):295-301.

35. Hull L, Lai MC, Baron-Cohen S, et al. Gender differences in self-reported camouflaging in autistic and non-autistic adults. Autism. 2020;24(2): 352-363.

36. Cage E, Di Monaco J, Newell V. Experiences of autism acceptance and mental health in autistic adults. J Autism Dev Disord. 2018;48(2):473-484.

37. Lai MC, Lombardo MV, Ruigrok AN, et al. Quantifying and exploring camouflaging in men and women with autism. Autism. 2017;21(6): 690-702.

38. Robinson E, Hull L, Petrides KV. Big five model and trait emotional intelligence in camouflaging behaviours in autism. Pers Individ Differ. 2020;152:109565.

39. Hull L, Petrides KV, Mandy W. Cognitive predictors of self-reported camouflaging in autistic adolescents. Autism Res. 2021;14(3):523-532.

40. Hull L, Levy L, Lai MC, et al. Is social camouflaging associated with anxiety and depression in autistic adults? Mol Autism. 2021;12:13.

41. Beck JS, Lundwall RA, Gabrielsen T, et al. Looking good but feeling bad: "camouflaging" behaviors and mental health in women with autistic traits. Autism. 2020;24(4):809-821.
42. Cassidy SA, Gould K, Townsend E, et al. Is camouflaging autistic traits associated with suicidal thoughts and behaviours? Expanding the interpersonal psychological theory of suicide in an undergraduate student sample. J Autism Dev Disord. 2020;50(10):3638-3648.

43. Dell'Osso L, Gesi C, Massimetti E, et al. Adult Autism Subthreshold Spectrum (AdAS Spectrum): validation of a questionnaire investigating subthreshold autism spectrum. Compr Psychiatry. 2017;73:61-83.

44. Donati MA, Berrocal C, Primi C, et al. Measuring subthreshold autistic traits in the general population: psychometric properties of the Adult Autism Subthreshold Spectrum (AdAS Spectrum) scale. Psychiatry Res. 2019;281:112576

45. Dell'Osso L, Carmassi C, Cremone IM, et al. Defining the optimal threshold scores for Adult Autism Subthreshold Spectrum (AdAS Spectrum) in clinical and general population. Clin Pract Epidemiol Ment Health. 2020; 16:204-211.

46. Dell'Osso L, Cremone IM, Muti D, et al. Validation of the Italian version of the Camouflaging Autistic Traits Questionnaire (CAT-Q). Compr Psychiatry. Submitted.

47. Dell'Osso L, Carpita B, Muti D, et al. Mood symptoms and suicidality across the autism spectrum. Compr Psychiatry. 2019;91:34-38.

48. Baron-Cohen S. The Essential Difference: The Truth About the Male and Female Brain. New York: Basic Books; 2003.

49. Hoekstra RA, Bartels M, Cath DC, Boomsma DI. Factor structure, reliability and criterion validity of the Autism-Spectrum Quotient (AQ): a study in Dutch population and patient groups. J Autism Dev Disord. 2008;38(8): 1555-1566.

50. Livingston LA, Happé F. Conceptualising compensation in neurodevelopmental disorders: reflections from autism spectrum disorder. Neurosci Biobehav Rev. 2017;80:729-742.

51. Stoet G, Geary DC. Gender differences in the pathways to higher education. Proc Natl Acad Sci USA. 2020;117(25):14073-14076.

52. Dell'Osso L, Carmassi C, Musetti L, et al. Lifetime mood symptoms and adult separation anxiety in patients with complicated grief and/or post-traumatic stress disorder: a preliminary report. Psychiatry Res. 2012;198(3):436-440.

53. Billeci L, Calderoni S, Conti E, et al. The broad autism (endo)phenotype: neurostructural and neurofunctional correlates in parents of individuals with autism spectrum disorders. Front Neurosci. 2016;10:346.

54. Dell'Osso L, Cremone IM, Carpita B, et al. Correlates of autistic traits among patients with borderline personality disorder. Compr Psychiatry. 2018;83:7-11.

55. Carmassi C, Corsi M, Bertelloni CA, et al. Mothers and fathers of children with epilepsy: gender differences in post-traumatic stress symptoms and correlations with mood spectrum symptoms. Neuropsychiatr Dis Treat. 2018;14:1371-1379. Published May 25, 2018. doi:10.2147/NDT.S158249.

56. Carmassi C, Palagini L, Caruso D, et al. Systematic review of sleep disturbances and circadian sleep desynchronization in autism spectrum disorder: toward an integrative model of a self-reinforcing loop. Front Psychiatry. 2019;10:366 